\title{
Solution of Maxwell's Equations for Cylindrical Symmetry Waveguides
}

\author{
N. V. Selina \\ Kuban State Technological University, Krasnodar, Russia \\ Email:Selina_Natalia@mail.ru
}

How to cite this paper: Selina, N.V. (2020) Solution of Maxwell's Equations for Cylindrical Symmetry Waveguides. Journal of Applied Mathematics and Physics, 8, 753-765. https://doi.org/10.4236/jamp.2020.85058

Received: March 23, 2020

Accepted: April 23, 2020

Published: April 26, 2020

Copyright $\odot 2020$ by author(s) and Scientific Research Publishing Inc. This work is licensed under the Creative Commons Attribution International License (CC BY 4.0).

http://creativecommons.org/licenses/by/4.0/

\begin{abstract}
The solution of Maxwell's equations for a piecewise homogeneous medium of cylindrical symmetry has been obtained. The parameters of the cylindrical waveguide modes have been calculated on its basis. The conclusions are confirmed by numerical calculation of the first four modes of a hollow metal waveguide operating as a mode convector.
\end{abstract}

\section{Keywords}

Maxwell's Equations, Circular Waveguide, Modes of the Waveguide

\section{Introduction}

Fiber-optic waveguides serve as a transfer medium for optical communication and information transmission systems. Over the past few decades, there has been a steady improvement in both the theory of waveguides [1] [2] and their manufacturing technologies with significant improvements in technical characteristics. The scope of their application is also expanding [1] [2] [3] [4] [5].

In addition to transmitting an optical signal over a distance, waveguides perform the functions of elements of integrated optoelectronic circuits [1] [2]. The physical and mathematical model of such a waveguide is defined by the term "planar". In it, the light wave propagates within the plane, that is, in two-dimensional space. The mathematical model of the propagation of an electromagnetic wave in such a structure is determined by an accurate calculation. A stronger localization of directed optical energy occurs in channeling waveguides, where the electromagnetic wave propagates in a single isolated direction. Such optical structures may have a circular, elliptical, or rectangular cross-section that corresponds to the selected direction. The waveguide has a middle part (core) closest to the symmetry axis, and a layer of the same shape covering the core (shell). There are 
hollow waveguides with a metal shell and dielectric waveguides, where the core is made of a material that is more optically dense than the shell material. In the latter structure, the direction of light in an optical fiber is carried out by the effect of full internal reflection at its borders. The hollow waveguide transmits the optical signal with minimal distortion. The shell can be metal or dielectric multilayer. Therefore, hollow structures can be used as a mod Converter [3] [4] [5].

The cylindrical waveguide supports modes that differ in radial and azimuthal mode numbers. An axisymmetric waveguide of any radius can excite an infinite number of modes with different azimuthal mode numbers. A larger waveguide will support many modes that differ in the number of zeros of the field in the cross section.

However, even in such a channel, it is possible to excite one mode if the conditions are such that the modes of high orders are strongly attenuated. In articles [3] [4] [5], the parameters of the modes of a cylindrical hollow waveguide with a metal shell are calculated. In the waveguide [4], all modes except the symmetrical transverse magnetic wave are suppressed. This waveguide is used as a mode Converter for high-power microwave sources. Its parameters are calculated in such a way as to highlight a single mode.

To solve such a problem, the Helmholtz equation is usually solved in a cylindrical coordinate system, and then the solutions corresponding to homogeneous regions are "stitched", according to the rule of continuity of the tangential components of the electromagnetic field at the media boundaries.

The Helmholtz equation in a cylindrical coordinate system has the structure of the Bessel equation. The order of the equation determines the azimuthal mode number. Thus, the solution is in this case special functions, operations on which are performed only by numerical methods, which complicates the procedure for calculating the dispersion diagram of the waveguide or, in the case of the inverse problem, the parameters of the waveguide corresponding to the desired characteristics of the electromagnetic wave.

In this article, a method for calculating the electromagnetic wave field in a cylindrical waveguide is proposed. The initial equations in it are the Maxwell equations, from which the wave equation (Helmholtz equation) is derived, taking into account the dependence of the solution on the piecewise constant permittivity. This results in a solution that is common to all components of a piecewise homogeneous medium.

Derived from Maxwell's material equations and Maxwell's differential equations, it satisfies the condition of continuity of the tangential components of the electromagnetic field at the interface of media. In the course of the problem, the Bessel equation was solved and its general integral was obtained, where the final solution depends on the coordinates and waveguide parameters by implicit definition via a transcendental equation that includes only elementary functions.

Using this method, the parameters of all waveguide modes are calculated. The essence of the method is a rule that allows you to write the integration 
constants of Maxwell's equations on all the interface boundaries medium of a layered structure. There is no need to "stitch" solutions of equations, since the continuity of tangential components of the field is a property of the General solution. The result of calculating the problem with the parameters defined in the article [4]. Result corresponds to the theoretical conclusions and data obtained in [4].

\section{Theoretical Solution of the Problem}

In order to obtain a general solution of Maxwell's equations, let's write down a wave equation derived from the system of Maxwell's equations excluding unknown functions-vectors of dielectric and magnetic induction, as well as the electric field strength:

$$
\begin{gathered}
\Delta \mathbf{H}+\frac{[\operatorname{grad}(\varepsilon) \times \operatorname{rot}(\mathbf{H})]}{\varepsilon}+\frac{\omega^{2}}{c^{2}} \varepsilon \mathbf{H}=0 \\
\frac{\omega^{2}}{c^{2}}=k_{0}^{2}
\end{gathered}
$$

where $\omega$ is the frequency of the electromagnetic wave and $\varepsilon$ is the permittivity of a two-component piecewise homogeneous medium:

$$
\begin{gathered}
\varepsilon(r)=\varepsilon_{1}+\left(\varepsilon_{2}-\varepsilon_{1}\right) T(r) \\
T(r)=\left\{\begin{array}{l}
1, \varepsilon(r)=\varepsilon_{2} \\
0, \varepsilon(r)=\varepsilon_{1}
\end{array}\right.
\end{gathered}
$$

$\left(\varepsilon_{1}, \mathcal{E}_{2}\right.$ is the permittivity of regions 1 and 2 respectively).

Since the interface between media with different permittivity has a cylindrical symmetry, let's calculate in a cylindrical coordinate system. Let's write down the equations that define the rotor and the vector product of vectors in cylindrical coordinates:

$$
\begin{gathered}
(\operatorname{rot} \mathbf{b})_{k}=\frac{\left(\frac{\partial\left(\boldsymbol{b}_{j} H_{j}\right)}{\partial x_{i}}-\frac{\partial\left(\boldsymbol{b}_{i} H_{i}\right)}{\partial x_{j}}\right)}{H_{i} H_{j}}, i, j, k=1,2,3 \\
{[\mathbf{a} \times \mathbf{b}]_{k}=\epsilon_{i j k} \boldsymbol{a}_{i} \boldsymbol{b}_{j},}
\end{gathered}
$$

here $\epsilon_{i j k}$ is a Levi-Civita symbol,

$$
\begin{aligned}
& x_{1}=r, x_{2}=\varphi, x_{3}=z, \\
& H_{1}=1, H_{2}=r, H_{3}=1
\end{aligned}
$$

We also use the following equation for cylindrical coordinates:

$$
(\Delta \mathbf{b})_{\varphi}=\Delta b_{\varphi}-\frac{b_{\varphi}}{r^{2}}+\frac{2}{r^{2}} \frac{\partial b_{r}}{\partial \varphi}
$$

First of all, let's define the vector product of the permittivity gradient on the magnetic field strength rotor. 


$$
\frac{[\operatorname{grad}(\varepsilon) \times \operatorname{rot}(\mathbf{H})]_{\varphi}}{\varepsilon}=-\frac{1}{\varepsilon} \frac{\partial \varepsilon}{\partial r} \cdot \operatorname{rot}(\mathbf{H})_{z}=-\frac{1}{\varepsilon} \frac{\partial \varepsilon}{\partial r}\left(\frac{1}{r} \frac{\partial\left(r H_{\varphi}\right)}{\partial r}-\frac{1}{r} \frac{\partial H_{r}}{\partial \varphi}\right)
$$

Let's consider two types of waves propagating in a cylindrical waveguide: transverse electric $\left(E_{z}=0\right)$ and transverse magnetic $\left(H_{z}=0\right)$ waves.

Thus, in a transverse magnetic wave, only the $\varphi$ - and $r$-components of the magnetic field strength differ from zero. For the $\varphi$-component, Equation (1) has the form:

$$
\Delta H_{\varphi}-\frac{1}{\varepsilon} \frac{1}{r} \frac{\partial \varepsilon}{\partial r}\left(\frac{1}{r} \frac{\partial\left(r H_{\varphi}\right)}{\partial r}-\frac{1}{r} \frac{\partial H_{r}}{\partial \varphi}\right)-\frac{H_{\varphi}}{r^{2}}+\frac{2}{r^{2}} \frac{\partial H_{r}}{\partial \varphi}=-H_{\varphi} \varepsilon k_{0}^{2}
$$

First of all, let's find the distribution of fields in a transverse magnetic wave. For the purpose of applying the method of separating variables, let's present the magnetic field strength function as a product of functions that depend on only one variable: $R(r)$ and $\Phi(\varphi)$.

Substituting this representation of the magnetic field strength in the wave equation, we transform it to the form:

$$
\begin{aligned}
& {\left[\frac{\Phi}{r} \frac{\mathrm{d}}{\mathrm{d} r}\left(r \frac{\mathrm{d} R}{\mathrm{~d} r}\right)+\frac{\Phi R}{r^{2}}-\frac{1}{\varepsilon} \frac{1}{r} \frac{\partial \varepsilon}{\partial r}\left(\Phi \frac{\mathrm{d}(r R)}{\mathrm{d} r}-\frac{\partial H_{r}}{\partial \varphi}\right)\right]} \\
& +\frac{R}{r^{2}} \frac{\mathrm{d}^{2} \Phi}{\mathrm{d} \varphi^{2}}+\frac{2}{r^{2}} \frac{\partial H_{r}}{\partial \varphi}=-R \Phi\left(\varepsilon k_{0}^{2}-k_{z}^{2}\right)
\end{aligned}
$$

The equation that defines the angular dependence of the field, let's define it as follows:

$$
\begin{gathered}
\frac{\mathrm{d}^{2} \Phi}{\mathrm{d} \varphi^{2}}=m^{2} \\
\Phi(\varphi)=\Phi_{0} \exp ( \pm i m \varphi)
\end{gathered}
$$

here $m$ is a wonderful mod number, $\Phi_{0}$ is a constant.

To apply the method of separating variables to Equation (2), let's define the relationship between the radial and azimuthal components of the magnetic field. To this end, let's write the dependence of the basic orts in the cylindrical coordinate system $\left(\mathbf{e}_{t}, \mathbf{e}_{\varphi}\right)$ and the cyclic orts $\left(\mathbf{e}_{1}, \mathbf{e}_{-1}\right)$, the field expansion of which determines the complex form of the electromagnetic field recording. The intermediate result contains the Cartesian unit vectors $\left(\mathbf{e}_{x x}, \mathbf{e}_{y}\right)$.

$$
\begin{aligned}
\mathbf{e}_{r} & =\cos (\varphi) \mathbf{e}_{x}+\sin (\varphi) \mathbf{e}_{y} \\
& =\cos (\varphi)\left(\mathbf{e}_{1}-\mathbf{e}_{-1}\right) / \sqrt{2}+\sin (\varphi)\left(\mathbf{e}_{1}+\mathbf{e}_{-1}\right) / i \sqrt{2} \\
& =\mathbf{e}_{1} \exp (-i \varphi) / \sqrt{2}-\mathbf{e}_{-1} \exp (i \varphi) / \sqrt{2} \\
\mathbf{e}_{\varphi}= & -\sin (\varphi) \mathbf{e}_{x}+\cos (\varphi) \mathbf{e}_{y} \\
= & -\sin (\varphi)\left(\mathbf{e}_{1}-\mathbf{e}_{-1}\right) / \sqrt{2}+\cos (\varphi)\left(\mathbf{e}_{1}+\mathbf{e}_{-1}\right) / i \sqrt{2} \\
= & -\mathbf{e}_{1} \exp (i \pi / 2-i \varphi) / \sqrt{2}+\mathbf{e}_{-1} \exp (i \varphi-i \pi / 2) / \sqrt{2}
\end{aligned}
$$

Thus, a transverse magnetic cylindrical wave can be represented as the sum of 
two components:

$$
\mathbf{H}=H_{r} \mathbf{e}_{r}+H_{\varphi} \mathbf{e}_{\varphi}=\frac{\left(H_{r}-i H_{\varphi}\right) \exp (-i \varphi) \mathbf{e}_{1}}{\sqrt{2}}-\frac{\left(H_{r}+i H_{\varphi}\right) \exp (i \varphi) \mathbf{e}_{-1}}{\sqrt{2}}
$$

Since Equation (1) is linear, the General solution is determined by a linear combination of two solutions for the component waves. A wave has only the first component if the equality is true:

$$
H_{r}=-i H_{\varphi}
$$

In this case, the polarization vector rotates relative to the $\mathrm{Z}$ axis in the direction of right rotation, which corresponds to the minus sign in the exponent (3). Then the last term in the left part of Equation (2) can be defined by the following relation:

$$
\frac{2}{r^{2}} \frac{\partial H_{r}}{\partial \varphi}=-\frac{2}{r^{2}} \frac{\partial i H_{\varphi}}{\partial \varphi}=-\frac{2 i R}{r^{2}} \frac{\partial \Phi_{-}}{\partial \varphi}=-m \frac{2 R \Phi_{-}}{r^{2}}
$$

For the wave component defined by the $\boldsymbol{e}_{-1}$ vector, the following equation is true:

$$
H_{r}=i H_{\varphi}
$$

Here there is a plus sign in the exponent (3), and the last term on the left side of Equation (2) is defined by the relation:

$$
\frac{2}{r^{2}} \frac{\partial H_{r}}{\partial \varphi}=\frac{2}{r^{2}} \frac{\partial i H_{\varphi}}{\partial \varphi}=\frac{2 i R}{r^{2}} \frac{\partial \Phi_{+}}{\partial \varphi}=-m \frac{2 R \Phi_{+}}{r^{2}}
$$

If we substitute expressions (4)-(5) in (2) and divide the equation by the azimuthal function $\Phi_{-}(\varphi)$ (or $\Phi_{+}(\varphi)$ ), we get the same equations for the radial function. That is, the field of the General transverse magnetic wave is determined by the General equation:

$$
\frac{1}{r} \frac{\mathrm{d}}{\mathrm{d} r}\left(r \frac{\mathrm{d} R}{\mathrm{~d} r}\right)-\left(1+2 m+m^{2}\right) \frac{R}{r^{2}}-\frac{1}{\varepsilon} \frac{1}{r} \frac{\mathrm{d} \varepsilon}{\mathrm{d} r}\left(\frac{\mathrm{d}(r R)}{\mathrm{d} r}+m R\right)=-R\left(\varepsilon k_{0}^{2}-k_{z}^{2}\right)
$$

The dependence on the $\mathrm{z}$ coordinate is determined by the multiplier $\exp \left(k_{z} z\right)$, where $k_{z}$ is the wave propagation constant. Assuming

$$
k_{r}^{2}=\varepsilon k_{0}^{2}-k_{z}^{2}
$$

let's transform Equation (6):

$$
\begin{aligned}
& \frac{1}{r} \frac{\mathrm{d}^{2}\left(R r^{-m}\right)}{\mathrm{d} r^{2}}+\frac{2 m+1}{r^{2}} \frac{\mathrm{d}\left(R r^{-m}\right)}{\mathrm{d} r}-\frac{1}{\varepsilon} \frac{1}{r} \frac{\mathrm{d} \varepsilon}{\mathrm{d} r} \frac{\mathrm{d}\left(R r^{-m}\right)}{\mathrm{d} r} \\
& -\frac{(2 m+1) r^{-m-2}}{\varepsilon} \frac{\mathrm{d} \varepsilon}{\mathrm{d} r} R=-R r^{-m-1} k_{r}^{2}
\end{aligned}
$$

For a piecewise homogeneous medium, the last term on the left side of this equation is the Dirac Delta function.

Note that the equality defined by the differentiation formula of the product of functions, known from the course of mathematical analysis, is valid:

$$
\frac{\mathrm{d}}{\mathrm{d} r}\left(\frac{1}{\varepsilon f} \frac{\mathrm{d} f}{\mathrm{~d} r}\right)=\frac{1}{f} \frac{\mathrm{d}}{\mathrm{d} r}\left(\frac{1}{\varepsilon} \frac{\mathrm{d} f}{\mathrm{~d} r}\right)-\varepsilon\left(\frac{1}{\varepsilon f} \frac{\mathrm{d} f}{\mathrm{~d} r}\right)^{2}
$$


Taking this into account, Equation (6) is converted to the following equality:

$$
\begin{aligned}
& \varepsilon \frac{\mathrm{d}}{\mathrm{d} r}\left(\frac{1}{\varepsilon r^{-m} R} \frac{\mathrm{d}\left(R r^{-m}\right)}{\mathrm{d} r}\right)+\frac{2 m+1}{R r^{-m-1}} \frac{\mathrm{d}\left(R r^{-m}\right)}{\mathrm{d} r} \\
& +\varepsilon^{2}\left(\frac{1}{\varepsilon r^{-m} R} \frac{\mathrm{d}\left(R r^{-m}\right)}{\mathrm{d} r}\right)^{2}-\frac{2 m+1}{\varepsilon} \frac{\mathrm{d} \varepsilon}{\mathrm{d} r}=-k_{r}^{2}
\end{aligned}
$$

Let's enter a notation:

$$
\frac{\varepsilon_{1}}{\varepsilon r^{-m} R} \frac{\mathrm{d}\left(R r^{-m}\right)}{\mathrm{d} r}=k_{1} \tan (a(r))
$$

using which, Equation (7) can be represented as:

$$
\begin{aligned}
& -\frac{\varepsilon_{1}}{\varepsilon} \frac{\mathrm{d}\left(k_{1} \tan (a(r))\right)}{\mathrm{d} r}-\frac{\varepsilon_{1}}{\varepsilon} \frac{(2 m+1) k_{1} \tan (a(r))}{r}+\left(k_{1} \tan (a(r))\right)^{2} \\
& =k_{1}^{2}-\Delta\left(k_{r}^{2} \frac{\varepsilon_{1}^{2}}{\varepsilon^{2}}\right)
\end{aligned}
$$

here $k_{1}$ is the radial component of the wave vector in the first medium (from the cylinder axis). Let's call the function $(r)$ a phase function. The equation is written for a homogeneous region with a permittivity $\mathcal{E}$ different from the permittivity of the first region $\varepsilon_{1}$, in which the term containing the Delta function $\left(\frac{\mathrm{d} \varepsilon}{\mathrm{d} r}\right)$ is equal to zero. In order not to lose the contribution of such a term, it is necessary to put on the borders of homogeneous regions: $\tan (a(d))=\infty$, $a(d)=\pi / 2 \quad(d$ is the radius of the border of the regions). The obtained condition coincides with the phase condition for propagation of the cylinder's own modes (the phase function takes into account the optical path of the wave and the phase change when the wave is reflected from the media boundary), so it is automatically taken into account when calculating the modes. First of all, let's find the solution of Equation (9) for the first area filled with a homogeneous medium, where the equality is true: $\varepsilon(r)=\varepsilon_{1}$ Considering the solution (8) of Equation (7), we find the derivative of the function $a(r)$ :

$$
\frac{\mathrm{d} a}{\mathrm{~d} r}=k_{1}-\frac{(2 m+1) k_{1}^{2} \tan (a(r))}{r\left[\left(k_{1} \tan (a(r))\right)^{2}+k_{1}^{2}\right]}=k_{1}-\frac{(2 m+1) \sin (2 a(r))}{2 r}
$$

let's define the desired function by another equality that differs from Equation (8) by changing the value of the phase function by a constant value $\pi / 2$ :

$$
\frac{\varepsilon_{1}}{\varepsilon r^{-m} R} \frac{\mathrm{d}\left(R r^{-m}\right)}{\mathrm{d} r}=k_{1} \cot \left(a_{1}(r)\right)
$$

Equation (7) in this notation has the form:

$$
\begin{aligned}
& \frac{\varepsilon_{1}}{\varepsilon} \frac{\mathrm{d}\left(k_{1} \cot \left(a_{1}(r)\right)\right)}{\mathrm{d} r}+\frac{\varepsilon_{1}}{\varepsilon} \frac{(2 m+1) k_{1} \cot \left(a_{1}(r)\right)}{r}+\left(k_{1} \cot \left(a_{1}(r)\right)\right)^{2} \\
& =k_{1}^{2}-\Delta\left(k_{r}^{2} \frac{\varepsilon_{1}^{2}}{\varepsilon^{2}}\right)
\end{aligned}
$$


For such a solution, the derivative of the phase (function $a_{1}(r)$ ) is equal to:

$$
\frac{\mathrm{d} a}{\mathrm{~d} r}=k_{1}+\frac{(2 m+1) k_{1}^{2} \cot \left(a_{1}(r)\right)}{r\left[\left(k_{1} \cot \left(a_{1}(r)\right)\right)^{2}+k_{1}^{2}\right]}=k_{1}+\frac{(2 m+1) \sin \left(2 a_{1}(r)\right)}{2 r}
$$

The solution of Equation (7) corresponding to the definition of function $\frac{\varepsilon_{1}}{\varepsilon r^{-m} R} \frac{\mathrm{d}\left(R r^{-m}\right)}{\mathrm{d} r}$

(8) is determined by integrating the logarithm derivative:

$$
R r^{-m}=\exp \left(\int k_{1} \tan (a(r)) \mathrm{d} r\right)
$$

Let's replace the sign of the variable and function $a(r)$ in Equation (10) and integrate it:

$$
a(-r)=k_{1}(-r)-\int_{0}^{-r} \frac{(2 m+1) \sin (2 a(-x))}{2 x}
$$

Assume that the function $a(r)$ is odd, and therefore the equality is true:

$$
a(-x)=a(x)
$$

Then Equation (14) coincides with Equation (9), and their solutions functions $a_{1}(r)$ and $a(\mathrm{r})$, provided they are odd, also coincide.

To calculate the function a, you must output the differentiation and integration rules for it. To do this, multiply Equations (10) (13) by $-\tan (a(r))$ and $\cot \left(a_{1}(r)\right)=\cot (a(r))$, respectively:

$$
\begin{gathered}
-\tan (a(r)) \mathrm{d} a=-\tan (a(r)) \mathrm{d} r+\frac{\cos (a(r))^{2}}{r} \mathrm{~d} r \\
\cot (a(r)) \mathrm{d} a=\cot (a(r)) \mathrm{d} r+\frac{\sin (a(r))^{2}}{r} \mathrm{~d} r
\end{gathered}
$$

Adding up the Equations ((13), (14)), we get the integration rule for the function $\cot (2 a(r))$ :

$$
\int 2 \cot (2 a(r)) \mathrm{d} a=\int 2 \cot (2 a(r)) \mathrm{d} r+\int \frac{2 m+1}{r} \mathrm{~d} r
$$

The integrand function is the solution of Equation (7) with the wave vector $2 k_{1}$. indeed, the sum of Equations (9) and (13) for the function $a=a_{1}$ is equation (13) for the function $2 \cot (2 a)$ and the value of the radial component of the wave vector $2 k_{1}$.

Using Equation (17), it is easy to calculate the final solution of Equation (6) in a homogeneous medium. Solutions of Equation (6) have the property: if two solutions with the wave vector $k_{r}$ satisfy the equation:

$$
\frac{1}{H_{\varphi 1}\left(k_{r} r\right)} \frac{\mathrm{d} H_{\varphi 1}\left(k_{r} r\right)}{\mathrm{d} r} \frac{1}{H_{\varphi 2}\left(k_{r} r\right)} \frac{\mathrm{d} H_{\varphi 2}\left(k_{r} r\right)}{\mathrm{d} r}=-k_{r}^{2}
$$

Then the following relation is true for them and for the solution of Equation (6) with the wave vector $2 k_{r}$

$$
H_{\varphi 1}\left(k_{r} r\right) H_{\varphi 2}\left(k_{r} r\right)=H_{\varphi}\left(2 k_{r} r\right)
$$


Then the following relation is true for them and for the solution of Equation (6) with the wave vector $2 k_{r}$ :

$$
\begin{aligned}
& \frac{H_{\varphi}\left(2 k_{r} r\right)}{r^{2 m}}=\text { const } \frac{\sin (2 a(r))}{r^{2 m+1}} \\
& H_{\varphi}\left(2 k_{r} r\right)=\text { const } \frac{\sin (2 a(r))}{r}
\end{aligned}
$$

Let's deduce the equation for the phase. For the purpose of defining the phase, let's first find an expression for the derivative of the function $\frac{\sin (2 a(r))}{r^{2 m+1}}$ defined by the equality (18):

$$
\frac{\mathrm{d}}{\mathrm{d} r}\left(\frac{\sin (2 a(r))}{r^{2 m+1}}\right)=2 k_{1} \cot (2 a) \exp \left(2 k_{1} \int_{0}^{r} \cot (2 a(x)) \mathrm{d} x\right)
$$

The integral in the exponent is already defined above and is equal to:

$$
2 k_{1} \int_{0}^{r} \cot (2 a(x)) \mathrm{d} x=2 k_{1} \int_{0}^{a(r)} \cot (2 a) \mathrm{d} a-(2 m+1) \ln (r)
$$

Therefore, the desired derivative is defined by the expression:

$$
\frac{\mathrm{d}}{\mathrm{d} r}\left(\frac{\sin (2 a(r))}{r^{2 m+1}}\right)=\frac{2 k_{1} \cos (2 a(r))}{r^{2 m+1}}
$$

Analyzing the structure of Equations (7) and (10), we note that the statement is true: if the function $H_{\varphi}=\frac{\sin (2 a(r))}{r}$ is the solution of equation (7), then the function $H_{\varphi}=\frac{\sin (2 a(r)+\pi / 2)}{r}$ will also be the solution of this equation, since the phase function in (5) is defined exactly up to a constant. For such a function, the formula is valid:

$$
\frac{\mathrm{d}}{\mathrm{d} r}\left(\frac{\cos (2 a(r))}{r^{2 m+1}}\right)=-\frac{2 k_{1} \sin (2 a(r))}{r^{2 m+1}}
$$

The analysis of the differentiation rules (18) (19), as well as the differentiation rules for the product of functions known from the course of mathematical analysis, leads to the following formulas:

$$
\begin{gathered}
\frac{\mathrm{d}}{\mathrm{d} r}\left(\frac{\cos (a(r))}{r^{m+1 / 2}}\right)=-\frac{k_{1} \sin (a(r))}{r^{m+1 / 2}} \\
\frac{\mathrm{d}}{\mathrm{d} r}\left(\frac{\sin (a(r))}{r^{m+1 / 2}}\right)=\frac{k_{1} \cos (a(r))}{r^{m+1 / 2}}
\end{gathered}
$$

Let's proceed to the calculation of the phase, the direct integral equation for which, obtained by integration (6), has the form:

$$
a(r)=k_{1} r-\int_{0}^{r} \frac{(2 m+1) \sin (2 a(x))}{2 x}
$$


The integral is calculated by applying the part-by-part integration formula $2 \mathrm{~m}$ +1 times using the equalities (20) (21).

$$
a(r)=k_{1} r-\sum_{n=0}^{m} \frac{(-1)^{n+1}(2 m+1) ! \cos (2 a(r))}{2\left(2 k_{1} r\right)^{2 n+1}(2 m+1-2 n) !}-\sum_{n=1}^{m} \frac{(-1)^{n+1}(2 m+1) ! \sin (2 a(r))}{2\left(2 k_{1} r\right)^{2 n}(2 m-2 n) !}
$$

As you can see, for $m>0$, the phase on the cylinder axis is zero, but for a mode with a zero azimuthal mode number, the phase at $r=0$ is not zero, and the field on the axis is infinite, which does not correspond to the physical meaning of the problem. In this case, you can perform integration using formulas (22) (23). The final result for a function $a(r)$ equal to zero on the cylinder axis has the form:

$$
a(r)=k_{1} r-\frac{\sin (a(r))^{2}}{2 k_{1} r}
$$

Having obtained the solution of the problem for a homogeneous medium, let's turn to the metal-dielectric structure. For a two-component piecewise homogeneous medium with cylindrical boundaries of the media-component interface, let's define the equality:

$$
\frac{k_{1} \varepsilon}{k_{r} \varepsilon_{1}}=\alpha(r)
$$

The magnetic field of the wave in this case is defined as:

$$
r^{-m} H_{\varphi}\left(2 k_{1} r\right)=H_{0} \exp \left(-\frac{\varepsilon}{\varepsilon_{1}} \int_{0}^{r} 2 k_{1} \cot (2 a(x)) \mathrm{d} x\right)
$$

In the notations corresponding to the multilayer structure, the transformation of the phase function is similar to the Equation (10):

$$
\frac{\mathrm{d} \tilde{a}}{\mathrm{~d} r}=k(r)-\frac{(2 m+1) k(r)^{2} \tan (\tilde{a}(r))}{r\left[(k(r) \tan (\tilde{a}(r)))^{2}+k(r)^{2}\right]}
$$

here

$$
\frac{\mathrm{d} \tilde{a}}{\mathrm{~d} r}=k(r)-\frac{(2 m+1) k(r)^{2} \tan (\tilde{a}(r))}{r\left[(k(r) \tan (\tilde{a}(r)))^{2}+k(r)^{2}\right]}
$$

$\left(k(r)\right.$-radial components of wave vectors in two media-components: $k_{1}$ or $k_{2}$ ). Integrating (24), we obtain the already familiar equation common to all media components, and determine the solution:

$$
H_{\varphi}\left(2 k_{r} r\right)=\frac{\sin (2 \tilde{a}(r))}{r}
$$

Now you can write an expression for the longitudinal and transverse components of the electric field in the wave. According to Maxwell's equations they are related to the magnetic field strength by the equations:

$$
i \varepsilon k_{0} E_{z}=\frac{1}{r} \frac{\partial}{\partial r}\left(r H_{\varphi}\right)-\frac{1}{r} \frac{\partial H_{r}}{\partial \varphi}
$$




$$
i \varepsilon k_{0} E_{r}=\frac{\partial H_{\varphi}}{\partial z}
$$

The solution of Equation (7) is continuous at the interface of media. Substituting into these equations the expression for the magnetic field strength $H_{\varphi}$, we make sure that this solution of Equation (7) automatically determines the continuity of the tangential component of the electric and magnetic fields at the interface of the media. The component of the electric field normal to the cylindrical interface of the media is transformed when changing the medium 1 to medium 2 according to the law $E_{r 1} \varepsilon_{1}=E_{r 2} \varepsilon_{2}$. The electric field strength is finite and different from zero on the cylinder axis.

In order to obtain a general solution of Maxwell's equations for a transverse electric wave, let's write a wave equation derived from the system of Maxwell's equations excluding unknown functions-vectors of dielectric and magnetic induction, as well as the magnetic field strength.

$$
\Delta \mathbf{E}+\frac{\omega^{2}}{c^{2}} \varepsilon \mathbf{E}=0
$$

In the case of a transverse electric wave, the radial component of the electric field $\left(E_{r}\right)$ satisfies the equation:

$$
\frac{1}{r} \frac{\mathrm{d}}{\mathrm{d} r}\left(r \frac{\mathrm{d} E_{r}}{\mathrm{~d} r}\right)-\frac{E_{r}}{r^{2}}-\frac{2}{r^{2}} \frac{\mathrm{d} E_{\varphi}}{\mathrm{d} \varphi}+\frac{1}{r^{2}} \frac{\mathrm{d}^{2} E_{r}}{\mathrm{~d} \varphi^{2}}+\frac{\mathrm{d}^{2} E_{r}}{\mathrm{~d} z^{2}}=-E_{r} \varepsilon k_{0}^{2}
$$

Here we used the formula:

$$
(\Delta \mathbf{b})_{r}=\Delta b_{r}-\frac{b_{r}}{r^{2}}-\frac{2}{r^{2}} \frac{\partial b_{\varphi}}{\partial \varphi}
$$

This equation is solved by the method applied to Equation (2) above. The solution looks like:

$$
E_{r}\left(2 k_{r} r\right)=\text { const } \frac{\sin (2 a(r))}{r}
$$

where the phase function $a(r)$, as before, satisfies Equation (17), and at the interface of media, the phase function is transformed by the law:

$$
\tilde{a}(r)=\arctan \left(\frac{k_{1}}{k(r)} \tan (a(r))\right)
$$

The functions of both components of the magnetic field of the transverse electric wave are continuous in all areas of the structure and at their borders. As in the case of a transverse magnetic wave normal component of the electric field is transformed when changing the medium 1 to medium 2 according to the law $E_{r 1} \varepsilon_{1}=E_{r 2} \varepsilon_{2}$.

\section{Numerical Calculation of Modes Parameters of a Cylindrical Waveguide Structure}

Equations (24) (25) can be used to calculate the parameters of the modes of a cylindrical waveguide of any material composition. If the waveguide is a pure 
channel in the region of a large volume (filled with metal) compared to the size of the channel, then the parameters of its own modes can be determined by studying a physical and mathematical model: a cylindrical cavity in an infinite metal space. High-power microwave mode Converter described in the article [4] is defined in this model. The elements of the waveguide scattering matrix of the first four modes of two different linear polarizations are calculated there. Waveguide of finite length $(250 \mathrm{~mm})$ with parameters: $45 \mathrm{~mm}$ waveguide radius, operating frequency $3 \mathrm{GHz}$. The data is taken from the article [4]. There is no attenuation of the TM01 mode, and other modes are significantly attenuated.

To calculate such a graph, you can also use a method based on the conclusions and formulas obtained in this article. Let's define the characteristic equation of an infinite two-component structure with the interface of media components of cylindrical symmetry. The wave energy is transmitted over a distance without loss if the field in the cross section of the waveguide does not penetrate the external environment. This condition is valid if the phase of the wave increases to a value having an infinitely large imaginary part during the transition to the external environment. Within the framework of the theoretical conclusions given above, taking into account (23), the mathematical equation of the considered condition has the form:

$$
\frac{k_{1}}{\varepsilon_{1}} \tan (a(d))=-\frac{k_{2}}{\varepsilon_{2}} i
$$

where $d$ is the radius of the waveguide, indices 1 and 2 refer, respectively, to the internal (cylinder) and external media. Here we have used the equality:

$$
\lim _{x \rightarrow \infty} \tan (i x)=i
$$

Using Equation (28), the equalities (24) (25) can be reduced to the form

1) For a zero azimuthal mode number:

$$
a(d)=k_{1} d-\frac{\left(\frac{k_{1}}{\varepsilon_{1}} \frac{\varepsilon_{2}}{k_{2}}\right)^{2}}{2 k_{1} d\left(1-\left(\frac{k_{1}}{\varepsilon_{1}} \frac{\varepsilon_{2}}{k_{2}}\right)^{2}\right)}
$$

2) For an azimuthal mode number equal to one:

$$
\begin{aligned}
a(d)= & k_{1} d+\frac{3\left(\left(\frac{k_{1}}{\varepsilon_{1}} \frac{\varepsilon_{2}}{k_{2}}\right)^{2}+1\right)}{4 k_{1} d\left(1-\left(\frac{k_{1}}{\varepsilon_{1}} \frac{\varepsilon_{2}}{k_{2}}\right)^{2}\right)}+\frac{3 i \frac{k_{1}}{\varepsilon_{1}} \frac{\varepsilon_{2}}{k_{2}}}{4\left(k_{1} d\right)^{2}\left(1-\left(\frac{k_{1}}{\varepsilon_{1}} \frac{\varepsilon_{2}}{k_{2}}\right)^{2}\right)} \\
& -\frac{3\left(\left(\frac{k_{1}}{\varepsilon_{1}} \frac{\varepsilon_{2}}{k_{2}}\right)^{2}+1\right)}{16\left(k_{1} d\right)^{3}\left(1-\left(\frac{k_{1}}{\varepsilon_{1}} \frac{\varepsilon_{2}}{k_{2}}\right)^{2}\right)}
\end{aligned}
$$




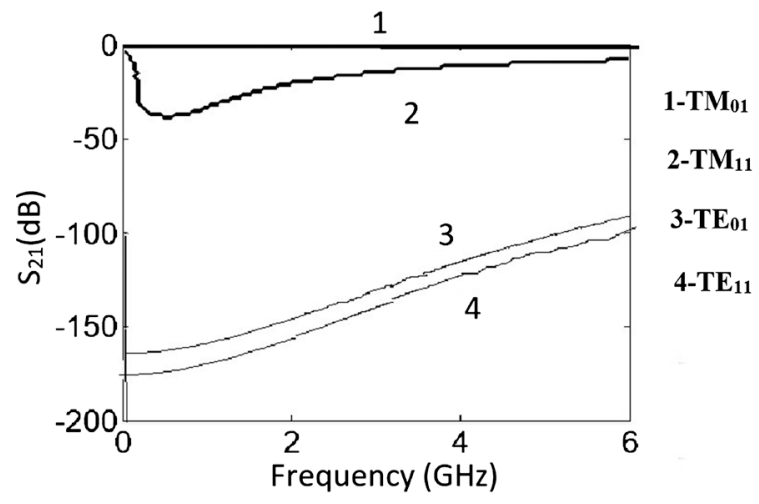

Figure 1. Cylindrical waveguide scattering parameter [4] for the first four modes.

The equation that determines the radial mode number $N$ :

$$
\operatorname{Re}\left(\arctan \left(\frac{k_{1}}{\varepsilon_{1}} \frac{\varepsilon_{2}}{k_{2}} \tan (a(d))\right)\right)=\frac{\pi N}{2}, N=1,2, \cdots
$$

The parameter of the scattering matrix S12 of a hollow metal waveguide calculated by the formulas (28)-(31) and similarly by formulas corresponding transverse electrical waves with the parameters specified in [4] is graphically presented in Figure 1. Analysis of graphs for the first four modes shows the advantage of $\mathrm{TM}_{01}$ mode propagation, since its attenuation coefficient is several orders of magnitude less than the attenuation coefficients of the other modes. This result confirms the fulfillment of the design condition reflected by the material and geometric parameters of the waveguide described in [4]. This indicates the reliability of the method based on theoretical conclusions and formulas presented in this article.

\section{Conclusion}

The method of calculating a waveguide with a round cross-section and a multilayer shell derived in the article allows us to write the solution of Maxwell's equations for such a structure with an arbitrary composition of media that fills the areas around the waveguide channel. In this solution, all tangential components of the electromagnetic field at the interface of the media are continuous. The method is based on the solution of the Bessel equation, which uses elementary functions, rather than special ones, as in the traditional solution of this problem. The original solution is more convenient for analyzing the dispersion properties of multilayer structures of cylindrical symmetry.

\section{Conflicts of Interest}

The author declares no conflicts of interest regarding the publication of this paper.

\section{References}

[1] Boudrioua, A. (2010) Photonic Waveguides: Theory and Applications. John Wiley 
\& Sons, Hoboken, 320 p. https://doi.org/10.1002/9780470611142

[2] Calvo, M.L. and Lakshminarayanan, V. (2007) Optical Waveguides: from Theory to Applied Mechanics. Taylor \& Francis Group, London,

[3] Jaiswal, R., Kumar, J., Singh, R. and Anitha, V.P. (2016) Simulation Investigation of TEM to TM01 Mode Conversion in Circular Waveguide. International Veda Conference, JNU, Delhi, 2016, 54-56.

[4] Kumar, V., Dwivedi1, S. and Jain, P.K. (2019) Circular Sectoral WaveguideTM01 to TE11 Mode Converter. Microwave and Optical Technology Letters, 61, 1697-1701, https://doi.org/10.1002/mop.31789

[5] Sobolev, D.I., Denisov, G.G., Eremeev, A.G., Holoptsev, V.V. and Tsvetkov, A.I. (2017) Polarization-Dependent TE11-to-TE11/TE01 Waveguide Mode Converter for Transmission Line Mode Switching, 10 th International Workshop 2017 "Strong Microwaves and Terahertz Waves: Sources and Applications" EPJ Web of Conferences, 149, 04017. https://doi.org/10.1051/epjconf/201714904017 